\title{
Mechanical properties of thin polysilicon films by means of probe microscopy
}

loannis Chasiotis, Wolfgang G. Knauss

loannis Chasiotis, Wolfgang G. Knauss, "Mechanical properties of thin polysilicon films by means of probe microscopy," Proc. SPIE 3512, Materials and Device Characterization in Micromachining, (1 September 1998); doi: 10.1117/12.324072

SDE Event: Micromachining and Microfabrication, 1998, Santa Clara, CA, United States 


\title{
Mechanical properties of thin polysilicon films by means of probe microscopy
}

\author{
Ioannis Chasiotis, Wolfgang G. Knauss \\ Graduate Aeronautical Laboratories, California Institute of Technology, Pasadena, CA 91125
}

\begin{abstract}
A new method for tensile testing of thin films is being developed. An electrostatic grip apparatus was designed and implemented to measure the elastic and ultimate tensile properties (Young's modulus, Poisson's ratio and tensile strength) of surface micromachined polysilicon specimens. The tensile specimens are "dog-bone" shaped ending in a large "paddle" for electrostatic gripping. The test section of the specimens is $400 \mu \mathrm{m}$ long and with $2 \mu \mathrm{mx} 50 \mu \mathrm{m}$ cross section. The method employs Atomic Force Microscope (AFM) or Scanning Tunneling Microscope (STM) acquired surface topologies of deforming specimens to determine (fields of) strains. By way of the method of Digital Image Correlation (DIC), the natural surface roughness features are used as distributed markers. The effect of markers artificially deposited on the surface is examined computationally. Also the significance of other parameters on property measurements, such as surface roughness, has been examined computationally. Initial results obtained using the tensile test apparatus are presented.
\end{abstract}

Keywords: Polysilicon, tensile test, mechanical properties, electrostatic grip, Atomic Force Microscopy, Digital Image Correlation, surface roughness, stiction.

\section{INTRODUCTION}

Polysilicon is the main structural material used in a wide variety of Microelectromechanical Systems (MEMS) fabricated by surface micromachining. Numerous applications of this technology with significant commercial impact have been developed in the form of sensors or actuators during the last ten years. The most common ${ }^{1}$ ones are micromotors, microgears, microvalves, springs, diaphragms, angular and resonant accelerometers, vibratory rate gyroscopes and many others. The reliability of MEMS devices is a major issue and it can only be addressed by direct measurements on small specimens with dimensions on the same order of magnitude as the fabricated microdevices. Such properties depend on the manufacture and process conditions, such as the type of substrate, deposition temperature, doping, annealing, chemical etching etc.

Various techniques ${ }^{2}$ have been developed in the past to address the issues of mechanical integrity and evaluation of the mechanical properties of polysilicon. Methods such as load-deflection, beam bending, tensile and nanoidentation tests, resonant frequency measurements of microcantilever beams have provided a span of values for the elastic constants of polysilicon. An important reason for the variety of given results is the large difference in the specimen geometries used. The load deflection method ${ }^{3,4}$ requires the knowledge of Poisson's ratio to evaluate Young's modulus and does not apply to compressively stressed films or to films that are difficult to make into membranes ${ }^{3}$. Moreover, since Poisson's ratio is unknown, the errors caused by assuming a value are magnified ${ }^{3}$ in evaluating Young's modulus. For example, for calculating the bending moment in beam bending tests two contributions must be taken into account; the rotation of the cantilever support point due to the applied force and the moment due to the stress gradient through the thickness of the cantilever. For this method the precise knowledge of the film thickness is mandatory to evaluate accurately the mechanical constants. In this context, surface roughness plays a potentially important role and, as will be shown later, contributes to the overall error.

Further author information -

W.G.K.(correspondence): Email: wgk@atlantis.caltech.edu; WWW: http://www.solids.caltech.edu/ wgk; Telephone: 626-395-4524; Fax 626-304-0175.

I.C.: Email: ichasiot@atlantis.caltech.edu; WWW: http://www.cco.caltech.edu/ ichasiot; Telephone: 626-395-4744. 
Tensile tests ${ }^{5-9}$, however, are less vulnerable to errors in geometry and are more reliable from an error analysis point of view. Tensile tests have been performed using either electrostatics to grip the specimens," adhesive media, ${ }^{5,6.8}$ or micromanipulators ${ }^{7}$ and specially designed specimens in a scanning electron microscope (SEM) ${ }^{7.9}$ or using interferometry ${ }^{5}$ to measure displacements. None of these techniques, however, measures the strains directly on the specimen. In the present technique, direct measurements on the specimen surface can be obtained with even atomic resolution. Also from the point of view of error analysis, tensile testing is the best way to measure the elastic modulus. In the present work, initial results are presented along with computational work to evaluate several parameters. The load-displacement curve has been obtained and by measuring the load cell stiffness and using a linear spring model, the elastic modulus is calculated.

\section{TESTING MICROTENSILE SPECIMENS}

\subsection{Test set-up}

The experimental arrangement for subjecting small "dog-bone" shaped specimens to tension, as shown in figure 1, is comprised of

1. a miniature load cell with an accuracy of $0.15 \%$ of full scale,

2. a computer controlled inchworm actuator to induce displacements to the specimen having a minimum step of $4 \mathrm{~nm}$,

3. a $y-z$ translation stage for positioning the sample. The local deformations of the specimen surface are monitored by means of an Atomic Force Microscope (AFM) (not shown in figure 1),

4. An additional measurement of the specimen deformation is obtained by the closed loop system that gives motion and controls the inchworm actuator, with an accuracy of $4 \mathrm{~nm}$ for every single movement of the actuator.

Dealing with small specimens demands the experimental device to be designed so that it simultaneously addresses several important issues, such as

1. specimen mounting,

2. electrical connections facilitation,

3. load cell and specimen alignment, and

4. proper fitting under the Atomic Force Microscope (AFM).

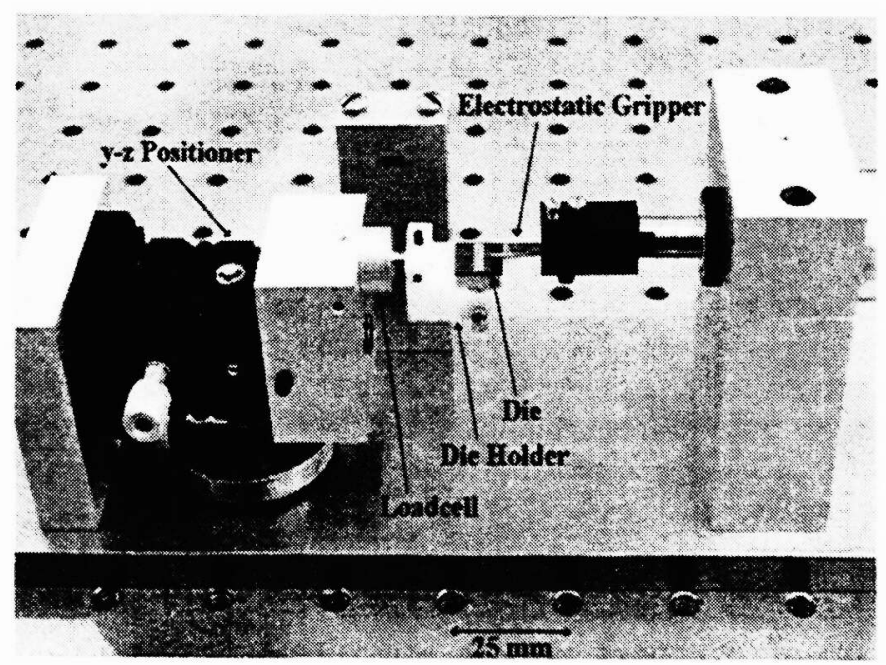

Fig. 1 The experimental setup for tensile tests

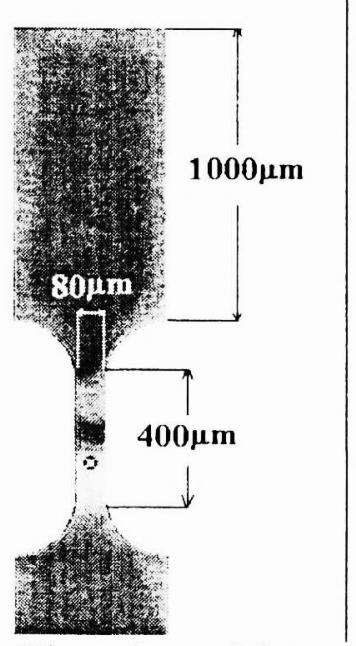

Fig. 2. Dimensions of the tensile specimens designed on a silicon chip, using the surface micromachining process. The thickness of the specimens is $2 \mu \mathrm{m}$.

The performance of each individual component has been examined for satisfactory accuracy. The hysterisis and linearity of the load cell have been evaluated to identify possible sources of error. The linearity for the loading part is very good for our purpose (error $0.10 \%$ ). The effective value of hysterisis is on the order of $1.8 \%$ of the maximum load, restricted only in the low load region, and it is constant and consistent over a sequence of several measurements. The resolution of the $y-z$ 
translation stage is on the order of 1 micron; this is sufficient so that proper positioning of the grip can be achieved as closely as possible to the specimen surface before applying the voltage. This capability prevents bending of the specimen during the test.

The accuracy of AFM has been tested in order to obtain a measure of the data repeatability. The Non-Contact Mode (NCM) was found to be a more accurate method than contact mode (AFM) for testing the specific type of thin film specimens, since vibrations may occur during the scan. On the other hand Non-Contact AFM is more suitable for surfaces that can readily develop a static charge.

The electrostatic grip is coated by a $200 \mathrm{~nm} \mathrm{Si}_{3} \mathrm{~N}_{4}$ insulating layer and was fabricated at the Electrical Engineering Department at Caltech. The very thin electrical insulation layer makes the necessary operational voltage relatively low. The electrical forces are sufficiently high to grip the specimen by applying a low potential of 10-20 Volts (since only the paddle's weight has to be overcome ${ }^{9}$ ). The operational voltage was on the order of $80 \mathrm{~V}$. The surface of the insulated grip is smooth with a topography variation of $10-20 \mathrm{~nm}$. This smoothness is the reason for the low values of friction coefficient that have been observed, since the contribution to this value results mostly from the roughness on the bottom surface of the specimen. The frictional force is derived from equation':

$$
F_{f}=-n \frac{\varepsilon_{0} \varepsilon}{2}\left(\frac{V}{d}\right)^{2} D L
$$

where $F_{\mathrm{f}}$ is the frictional force, $\mathrm{n}$ is the friction coefficient between the two surfaces, $\varepsilon_{0}$ and $\varepsilon$ are the dielectric constants of vacuum and silicon nitride $\left(10^{7} \mathrm{~V} / \mathrm{cm}\right)$ respectively, $d$ is the silicon nitride insulation thickness, $D, L$ are the width and length of paddle respectively. This force and, in addition, the electrical force exerted in a direction parallel to the paddle (which is minimal compared to the friction) contribute to the total force that grips the specimen.

\subsection{Specimen design}

Tensile, "bone-shaped" specimens with test section dimensions of $400 \times 80 \times 2$ microns and $400 \times 50 \times 2$ microns (figure 2 ), attached to a silicon wafer (figure 3), have been designed. They are free standing beams ending in a large paddle for electrostatic gripping. The deformation of the test section area is to be monitored by an AFM in two modes of operation. The first employs four thin gold markers $20 \mu \mathrm{mx} 5 \mu \mathrm{m}$ to define a square area (figure 4), opposing sides of which serve as base length markers of "micro-strain gages". Two different types of specimens (with and without etch holes) were designed for two different runs at the Microelectronics Center of North Carolina (MCNC), and are referred to as MUMPs19 (without etch holes) and MUMPs21 (with etch holes); they are illustrated in figure 2 . The large paddle of the specimen rests on the substrate; this can be determined from specimen on the right from Newton's fringes which, however, do not show up clearly at this scale in the figure. The fact that the specimens rest on the substrate- due to their "large dimensions"- led to stiction after their release. This stiction demanded special treatment before testing the specimens as discussed later.

The specimens from MUMPs2 1 were designed so as to take into account spatially frequent use of etch holes to facilitate the post-processing stage of oxide etching. The pattern for the paddle etch holes was chosen to avoid specimen fracture from any stress concentrations in the transition from the convergent section to the test section. Another point that has been addressed is the use of an "anchor" for the large paddle grip section. This ensures fracture-free handling before testing and decreases the risk of (potentially disastrous) specimen drift on the silicon wafer during the releasing process. A special probe stage has been built to cut the narrow beam that serves as an anchor for the paddle.

\subsection{Specimen manufacturing process}

The Multi-User MEMS Process (MUMP) has been used to manufacture the specimens. It is a surface micromachining process that uses three polysilicon layers as structural layers. A silicon wafer with (100) orientation, heavily doped with phosphorus using $\mathrm{POCl}_{3}$ is used as the substrate. A $600 \mathrm{~nm}$ electrical insulation layer of low-stress silicon nitride layer follows, deposited by low pressure chemical vapor deposition (LPCVD). This layer is essentially a hard insulation for the structures from the substrate. Then the first (nonstructural) layer of polysilicon is deposited for 500nm by LPCVD and photolithographically patterned by exposing a photoresistive material with the appropriate mask. The photoresist is etched 
away by a Reactive Ion Etch (RIE). This is followed by a $2.0 \mu \mathrm{m}$ phosphosilicate glass (PSG) LPCVD deposition. The latter is a sacrificial layer and by being lithographically patterned, can provide dimples under the next polysilicon film.

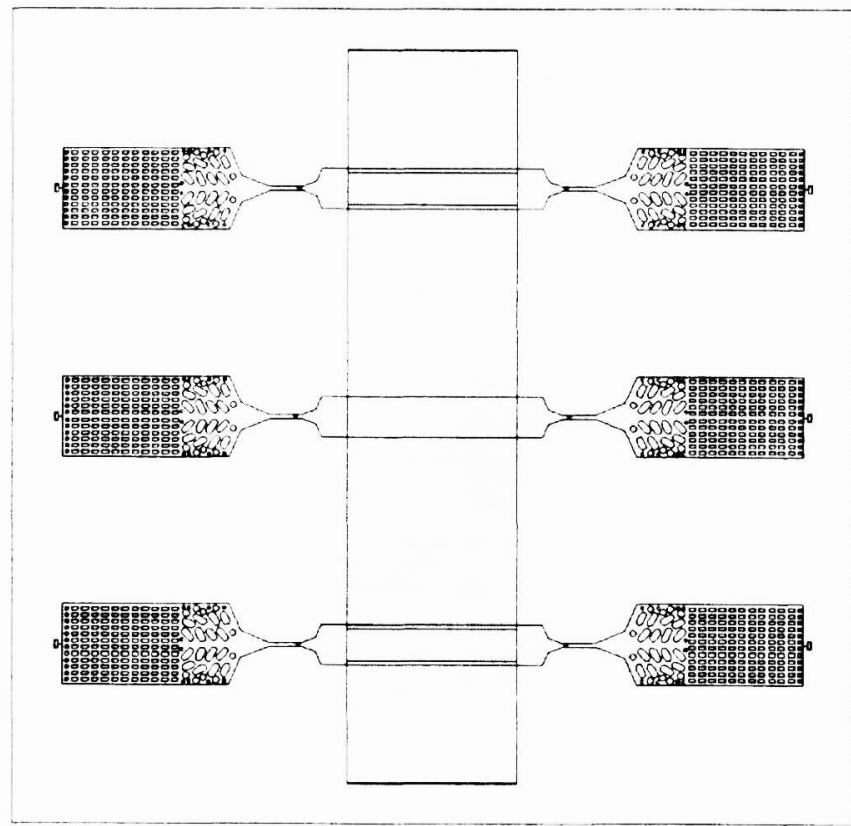

Fig. 3 Schematic of the wafer design with six free standing beams from MUMPs21. The dimensions of the die is $1 \mathrm{~cm} \times 1 \mathrm{~cm}$

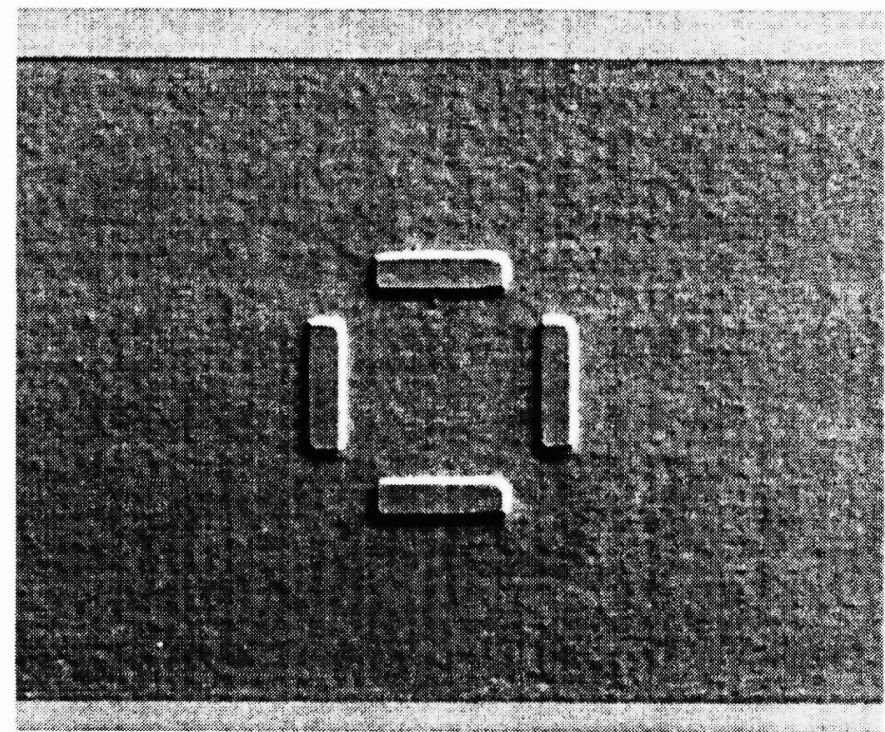

Fig. 4 The marked $30 \times 30 \mu \mathrm{m}$ area on the specimen surface. The marks are four thin film gold bars bonded to the surface with a thin chrome adhesion layer.

A mask is used to etch holes in the PSG and provide anchor holes to be filled by the subsequent polysilicon layer. Then a $2.0 \mu \mathrm{m}$ thick polysilicon structural layer is deposited followed by a $200 \mathrm{~nm}$ PSG layer and annealing at $1050^{\circ} \mathrm{C}$ for 1 hour. This provides uniform doping to the middle polysilicon layer and reduces the residual stresses in the structural layer. The latter and the PSG masking layer are lithographically patterned using a mask to define the structures built by the first structural layer. Then the PSG layer is etched and used as a resistant hard mask to the etchant for the subsequent polysilicon etch providing more accurate patterning. This hard oxide mask is removed by RIE after the polysilicon has been etched. Finally, for the marker metalization the wafer is patterned lithographically with a mask and a $0.5 \mu \mathrm{m}$ metal layer of gold with a thin chrome adhesion layer, is deposited and patterned. The photoresist and the unwanted metal are then removed in a solvent bath. Next the removal of the sacrificial layer and release of the structures is performed by immersing the chip in a bath of $49 \% \mathrm{HF}$ for a sufficient amount of time that depends on the size of the structures and the density of the etch holes. This is followed by rinsing with de-ionized water and then alcohol to reduce stiction. Then the dies are baked for 10 minutes at $110^{\circ} \mathrm{C}$. The optional $\mathrm{CO}_{2}$ supercritical process can be used instead of the last step of baking the wafer, in order to reduce the stiction danger further.

\subsection{Specimen release}

We have recently successfully released specimens at Caltech that suffered from stiction (MUMPs19). The release time needed in addition to the 2.5 minutes of incomplete release performed by MCNC, was another 13.5 minutes in $49 \% \mathrm{HF}$ with 15 minutes rinsing in water, 35 minutes post treatment in methanol in order to minimize stiction, and 5 minutes of oven baking at $100^{\circ} \mathrm{C}$. During the release the polysilicon cantilever beams became strongly curved up in the water and subsequently in the alcohol, but finally they remained stuck to the wafer surface. The bending is attributed to the high compressive stresses in the polysilicon as a result of the prior deposition on silicon oxide and of the mismatch of the thermal expansion coefficient.

The first problem encountered was the stiction of the specimens. The electrostatic forces needed for lifting the specimen paddle and bringing it in contact with the grip proved to be insufficient to overcome the stiction forces. Although these forces should not be so strong theoretically, they were strong enough to prevent pulling the paddle off from the wafer. ${ }^{10}$. 11 The 
adhesion forces are strong enough to overcome the action of the restoring elastic forces and thus the beams cannot be freed from the substrate. Several types of forces that appear in the interface contribute to this phenomenon; depending on the separation of the two surfaces, their intensity varies, but in general they are the capillary, electrostatic, van der Waals, solid bridging and hydrogen bonding forces ${ }^{10}$. Several solutions ${ }^{11}$ have been proposed to avoid stiction, but when the problem arose we found that the only solution was to nudge the structures mechanically. The long HF treatment has deteriorated the quality of the specimens and the low fracture limit indicates that HF may have attacked the grain boundaries (see also the paragraph on test results) leading to early fracture or fracture at cross sections other than the test section (see figure 6). Another way had to be determined to free the structures and this was performed by probing them mechanically with a fine tool tip. To this end a very sensitive and accurate device ending in a very sharp tip was used, so that the movement of the tip would not fracture the specimens. The tip was manufactured using the same technique that is used to fabricate sharp tips for STM devices by subjecting a Tungsten wire to $\mathrm{NaOH}$ solution and applying a suitable voltage.

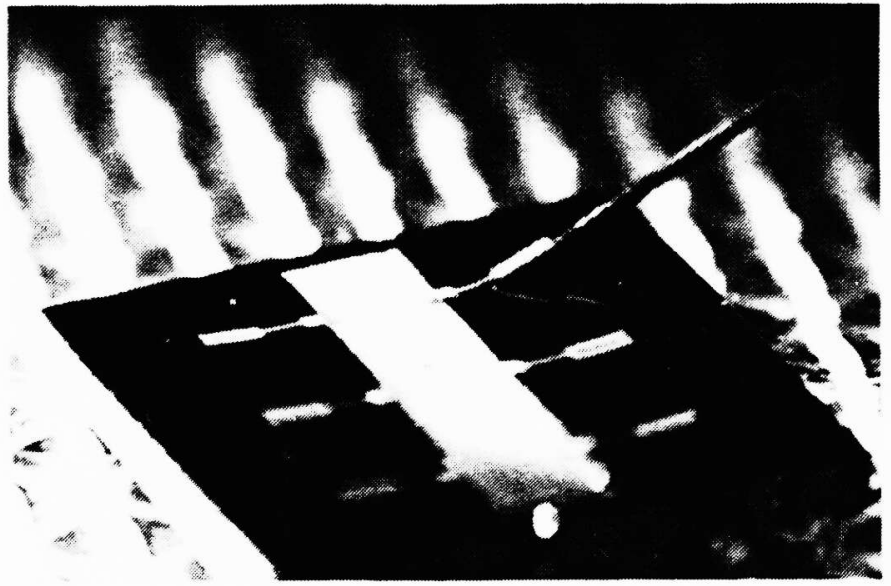

Fig. 5. The stiction problem was an important issue; the release was accomplished by mechanically forcing the specimen paddle end up from the substrate.

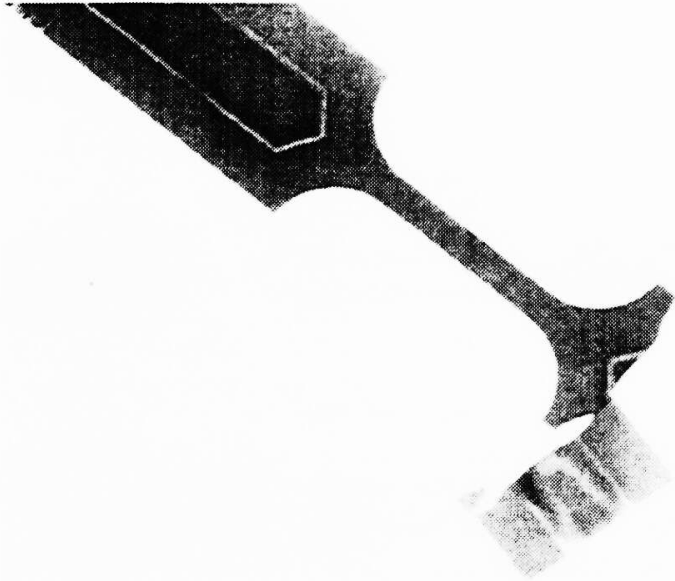

Fig. 6. Specimen fracture during test. The fracture occurred in an area that had been affected by the (necessary) second etching

We have recently received completely etched specimens from MCNC (free specimens, held only at one end and by way of the "anchor") after 11.5 minutes HF release employing the supercritical $\mathrm{CO}_{2}$ process to eliminate the (potential) problem of "stiction". These specimens correspond to the design for the MUMPs21 (see figure 2) and will be tested to compare with specimens from MUMPs19.

\subsection{Specimen surface topography}

The mechanical behavior of LPCVD manufactured thin films depends on various process parameters that affect the geometrical and physical characteristics of the film. A thorough investigation can be found in literature tracing parameters ${ }^{12}$ such as the residual stresses, film thickness, high temperature annealing, doping, etc. A geometrical parameter, which is the result of the deposition conditions and subsequent doping, is the surface roughness. This has to be taken into account especially when evaluating bending test data or performing tests that involve out-of-plane deformation. This surface roughness has been identified as an important reason for the scatter of the results in the strength measurements and cracking is assumed to initiate at surface defects ${ }^{7}$ or depend on the particular grain size ${ }^{8}$.

For the present material the surface topography appears to be uneven with columnar grains (figure 7 right) and an average grain size of $290 \mathrm{~nm}$ diameter with a high distribution of values ranging in a Gaussian distribution from $200 \mathrm{~nm}$ to $400 \mathrm{~nm}$ (figure 7 left). The bumps that are not overlapping are considered to be grains separated by grooves associated with grain boundaries. Measuring the polysilicon film thickness variation using an AFM, the ratio of the surface roughness to the specimen thickness is on the order of $1-2.5 \%$. The height of the bumps follows also a Gaussian distribution with maximum probability $50 \mathrm{~nm}$ and $45 \mathrm{~nm}$ for MUMPs 19 and MUMPs2 1 respectively. The minimum and maximum values of roughness are 30 and 60 for MUMPs 19 and 25 and 60 for MUMPs21. So the roughness appears to be consistent between different runs. However these values differ slightly after exposing the specimens to HF. X-ray diffraction measurements ${ }^{13}$ revealed that layers with compressive residual stresses are strongly dominated by (110) orientation since the grains from LPCVD grow 
faster in the $\langle 110\rangle$ crystallographic directions, displaying a columnar structure ${ }^{7.13}$. Layers with tensile stress have displayed additional (100) and to a lesser extent (111) structure ${ }^{7.13}$. Thus, the expected values for Young's modulus must be in the range of $131 \mathrm{GPa}$ to $189 \mathrm{GPa}$. The topography varies with grains protruding up to $30 \mathrm{~nm}$ above the average level of the surface. These are certainly parameters that have to be taken into account when the experimental results are evaluated. A computational evaluation of the effects of these features has been performed to provide a better interpretation of the experimental data.
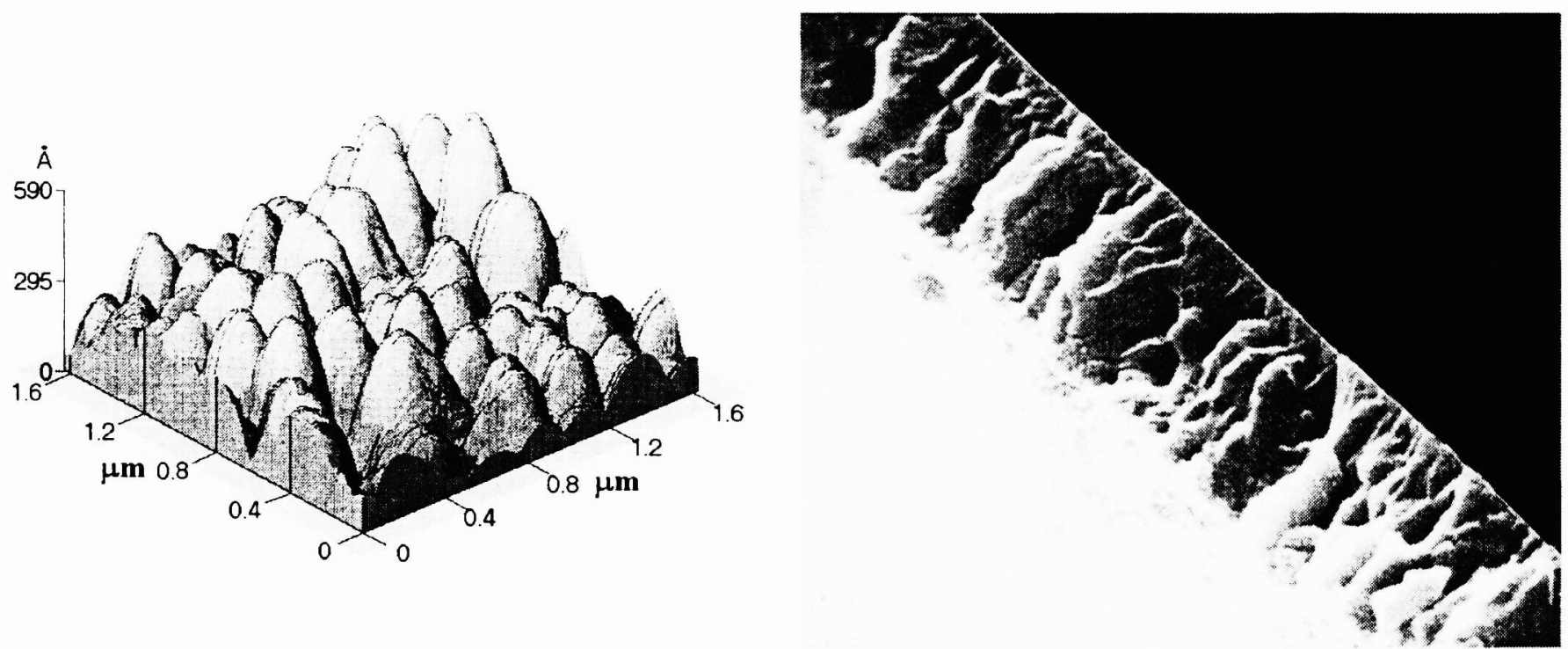

Fig. 7 Left: Three-dimensional rendering of the surface topography of a $1.6 \mu \mathrm{m} \times 1.6 \mu \mathrm{m}$ region revealing the columnar structure of the material and the surface non-uniformity. The z-direction is exaggerated compared to the lateral dimensions to make the grain structure more apparent. The maximum value on the $z$-axis is the local maximum height value. The image was obtained using an AFM in the Contact Mode. Right: SEM picture of the fractured cross section of the specimen. The columnar grain structure can be seen from the grooves and the extrusions that characterize the fractured section.

\subsection{Film thickness measurements}

The AFM is capable of providing images with atomic resolution. This assures that AFM measurements provide an accurate value of the film thickness, provided the latter has a thickness within the capability range of the microscope. The thickness that was so measured for MUMPs 19 polysilicon was $1895 \mathrm{~nm}$ with a std. deviation of 50 , compared to the value given by MCNC (using a different method) of $1965 \mathrm{~nm}$, with a std. deviation of 48 . The AFM value agrees also with the value obtained by means of an SEM $(1880 \mathrm{~nm})$. The thickness that was measured for MUMPs21 for polysilicon was $1915 \mathrm{~nm}$ with a std. deviation $40 \mathrm{~nm}$, compared to the given value of $1926 \mathrm{~nm}$, with a std. deviation of $42 \mathrm{~nm}$. Basically the standard deviation corresponds to the surface roughness we have analysed in the previous paragraph. The two independent measurements (by means of AFM and SEM) gave almost the same results that can be used to obtain more accurate values of the elastic constants than by using the nominal value $(2 \mu \mathrm{m})$ for the film thickness. For example, using the nominal value of the thickness for the case of tensile test it yields a $5 \%$ error that translates into $12 \%$ for bending.

\subsection{First test results}

Having learned to release the specimens from the substrate they were tested using the electrostatic grip. Due to the fact that the available batch of the specimens was etched twice, the fracture location was not in the test section (figure 6). The fracture was brittle, as expected, but occurred at a location that has been affected by the repeated HF release and the extended etching time. As seen in figure 6 , the partial release and the long time rest of the specimen caused a possible thinning along the bright wedge shaped line on the paddle. Similar results have been reported in the literature for various treatments ${ }^{14}$ with hydrofluoric acid that resulted in variations in Young's modulus and failure strength. On the one hand, long exposure to HF causes surface smoothing, but HF also attacks the grain boundaries to weaken the film surface, on the other. 


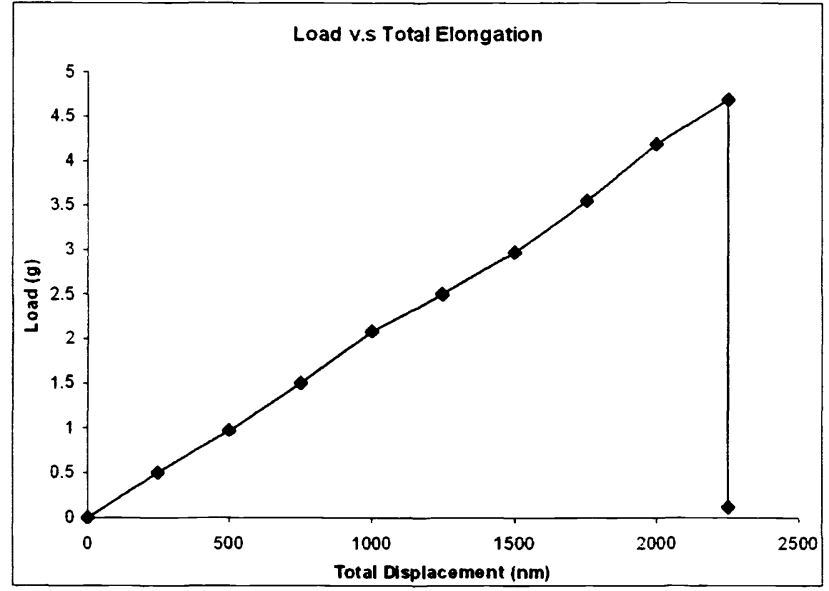

Fig. 8. Load v.s total elongation of the specimen.

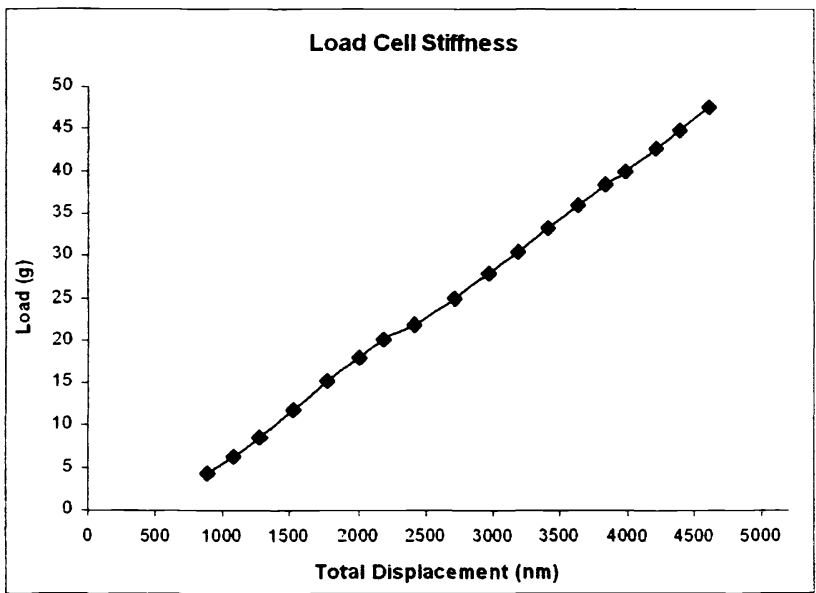

Fig. 9. Load v.s elongation of load cell.

The force-elongation behavior is shown in figure 8 . The voltage used to test these structures was 80 Volts. Higher voltages led to sparking and specimen damage. For lower voltage the linear behavior is modified by steps that indicate slipping between the grip and the paddle, resulting in intermittently stable gripping. The higher voltages gave stable behavior until fracture, since no slip occurred during a test. The system stiffness has been also measured at the end of the test mostly comprised by the stiffness of the load cell. The contribution of the rest parts of the test system to the overall compliance is small compared to that of the load cell.

This work is part of that "Round-Robin" experimental determination of physical properties of polysilicon specimens ${ }^{2}$ at the micron scale. Because of a considerable range of uncertainties in the determination of physical properties at the micron and nanoscale the objective of the present project was to perform a comparative study of modulus and possibly fracture behavior of polysilicon specimens by means of different experimental methods. Participants in this effort were Prof. W.N. Sharpe [Johns Hopkins University], S. Brown [Failure Analysis Associates] and Prof. G.C Johnson [U.C Berkeley]. The specimens were all manufactured in the same runs by the Microelectronics Center of North Carolina (MCNC). The results of the participants are presented at table 1 along with the present ones.

\begin{tabular}{|l|c|c|c|c|}
\hline \multicolumn{5}{|c|}{ Table 1. Round Robin results } \\
\cline { 2 - 5 } & U.C Berkeley & $\begin{array}{c}\text { Failure Analysis } \\
\text { Associates }\end{array}$ & $\begin{array}{c}\text { Johns Hopkins } \\
\text { University }\end{array}$ & Caltech \\
\cline { 2 - 5 } & 2 & 2 & $1.5 / 3.5$ & 1.89 \\
\hline Thickness (microns) & 9 & 2.5 & 9 & $2.5+13.5$ \\
\hline Etch time (minutes) & 174 & 137 & $136 / 142$ & 132 \\
\hline Young's modulus (GPa) & 2.8 & 2.7 & 1.3 & \\
\hline Fracture Strength (GPa) & & & & \\
\hline
\end{tabular}

The difference in the values we present is probably the result of the long etching time that weakened the specimen structure. The specimens, as mentioned, broke at the weak region (figure 6) which was possibly thinner. The measured fracture strength was $0.3 \mathrm{GPa}$, which is a low limit for strength of the test section, and is definitely higher in reality. In conclusion we see that the value that is mostly affected by the long HF exposure was the fracture strength, as expected, since the geometric characteristics of the specimen changed displaying a non-uniform thickness. 


\section{THEORETICAL AND COMPUTATIONAL WORK}

\subsection{Effect of markers on the specimen}

As mentioned before, we employ four thin metal markers ${ }^{5.6}$ deposited on the specimen test section with dimensions $20 \mu \mathrm{m} \times 5 \mu \mathrm{m}$ arranged in a square to define "micro-strain gages" (figure 4). In this mode of operation the AFM is used to determine the distance changes between the markers for measuring strains in the tension and transverse directions. A finite element program was developed using ABAQUS to investigate the influence of the gold markers deposited on the specimen surface on the stress and displacement field. A plane stress model has been used. The displacement field around the markers and in the surrounded area does not seem to be significantly affected by them. However they do introduce significant bending due to the much lower elastic constants and the much higher Poisson's ratio for gold (figure 10). The contrast of the markers represents their top surface strain field rather than the strains on the specimen's surface. The mismatch of the elastic moduli between polysilicon and gold results in bending of the metal marks. The strains in the direction of the applied force locally exceed $20 \%$ of the far field values. The FEM analysis can be used along with the experimentally obtained strain data to accurately evaluate the strain field through the application of the DIC method.

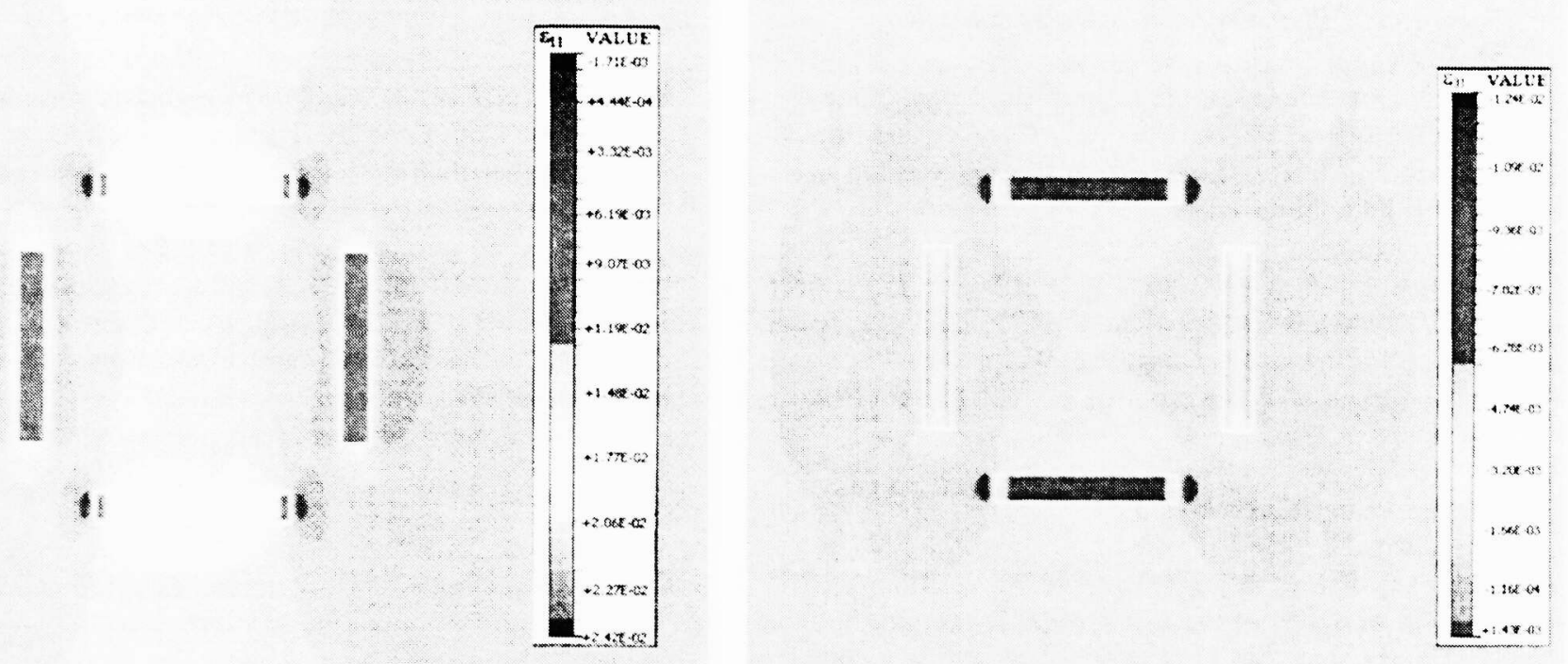

Fig. 10. $\varepsilon_{11}$ and $\varepsilon_{33}$ strain field around the gold markers. The strain field appears to be slightly distorted.

\subsection{Effect of surface roughness}

Accurate thickness measurements are important in order to evaluate mechanical properties. The uncertainty of the thickness of the film is increased by the fact that the surface displays roughness due to the emerging grains (small amplitude roughness) and micro twinning ${ }^{13}$ (large amplitude). LPCVD deposition and lithographic methods inherently cause an uneven surface topography. Usually, this feature is not taken into account when evaluating experimental results. A (two-dimensional) finite element program (ABAQUS) has been exercised to investigate the influence of surface roughness (bumps) as observed in the micro-manufactured specimens. A plane stress model is used. The influence of two parameters was examined: The average bump height (wave amplitude) and the bump width (wavelength) for both tensile and bending tests. The bumps have been modeled as sinusoidal surface variations, to represent the real surface topography (see also figure 7 left) of the AFM data.

The general sensitivity of the data surface roughness is shown in figures 11 and 12 for the tension and bending cases. We hasten to point out that these results are two-dimensional, and that three-dimensional roughness are expected to lead to wider variations. The parameter range used for the computations is larger than that usually observed, and was chosen to verify the data tendency. A very refined mesh is needed to obtain convergence for very small amplitude values. 
In general, indirect test methods are based on approximate values of the specimen thickness. This numerical analysis proves that the error due to the surface roughness of these approximations can be important, sometimes exceeding the experimental and systematic errors. When one speaks of surface roughness one may also mean the roughness on a single grain; we believe that contribution from this kind of roughness is minimal compared to that discussed above. This noise amplitude is on the order of a few nanometers.

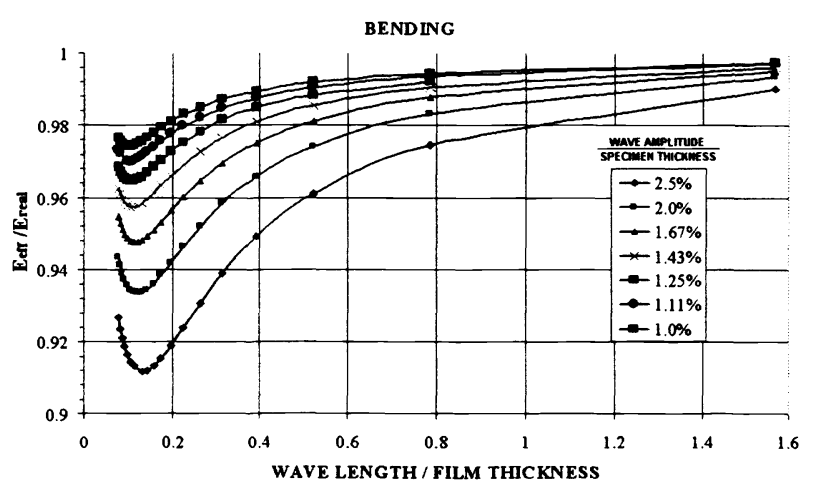

Fig. 11. Dependence of the ratio of the actual Young's Modulus to the "effective value", $\mathrm{E}_{\text {eff }} / \mathrm{E}_{\text {real }}$, measured in a bending test with respect to the wavelength and the amplitude of the surface bumps.

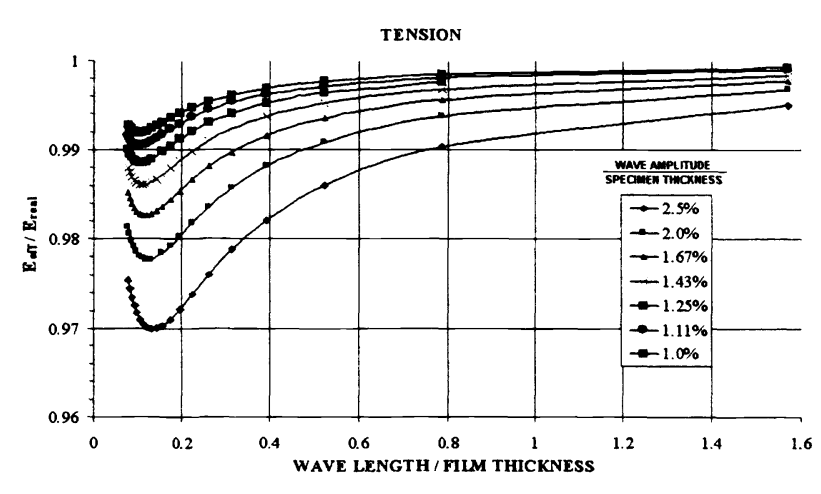

Fig. 12. Effect of surface roughness on tensile properties. Compared to figure 11 the effect is three times smaller than the effect on bending and minimal compared to other experimental errors.

Comparing the two plots (figure 11 and figure 12) it becomes obvious that the effect of the surface roughness to the data accuracy is stronger in the case of bending tests, reaching values of almost $10 \%$ for small grain diameters and high amplitudes. Taking into account the data provided in figure $7 \mathrm{left}$, according to the plots in figure 11 and figure 12 , the error for the tensile test is on the order of $1.5 \%$, while for bending the error is almost $5 \%$.

\subsection{Digital Image Correlation}

As mentioned before, a Digital Image Correlation (DIC) ${ }^{15-19}$ method resolves strains through an interpolation scheme. The deformation of a part of the test section is monitored by an AFM in two modes of operation. As discussed before, the first mode employs four thin gold markers to define a square area, opposing sides of which serve to define the base length of "micro-strain gages". In this mode of operation the AFM is used to determine the length changes between the markers. The second mode of operation does not employ such markings, but, by way of the method of Digital Image Correlation (DIC), makes use of the natural surface roughness features as distributed markers. In this operation mode the base length for strain determination is considerably smaller. This method of extracting in-plane deformations requires the ability to follow shape changes by comparing (surface) records before and after deformation. This information is obtained by a probe microscope (AFM) through records of surface features taken before and after deformation, and then computing from this information the movement of the (three-dimensional) surface features. Digital Image Correlation achieves this by defining a correlation coefficient as a function of the kinematic transformation relating the deformed to the undeformed geometry. The strains and displacements are parameters in this mapping, and are determined by a (nonlinear, multi-degree of freedom) optimization of the correlation coefficient. The method is capable of detecting displacements with a resolution of about $1 / 8$ of a pixel, which implies the capability of resolution strains on the order $0.03 \%-0.05 \%$.

\section{SUMMARY - FUTURE WORK}

The release of the specimens' free end has turned out to be more difficult than anticipated because of the large dimensions of the structures. After this problem was resolved, the force-displacement response of the very small specimens was successfully recorded. After adjustments to force-deformation data to allow for the compliance of the load cell, and a numerical evaluation of the deformed geometry, the material modulus was obtained. The fracture strength results are smaller than the values obtained by the other members of the Round Robin program which can possibly be attributed to the quality of 
the specimens after being exposed to HF for long time. Following this accomplishment the second mode of operation of the AFM will be pursued to determine the strain directly from scanning the surface of specimens from both MUMPs19 and MUMPs21 runs while under load and by implementing the DIC analysis. Ultimately this mode of operation is the preferred version.

\section{ACKNOWLEDGMENTS}

The authors would like to acknowledge Dr. Y. C. Tai of Electrical Engineering Department at Caltech and his graduate student Xing Yang for their help in releasing process. This work has been supported in part by the Airforce Office of Scientific Research (Round Robin Program) through grant F 49629-97-1-0324, under the direction of Major Brian Sanders.

\section{REFERENCES}

1. R. T. Howe, B. E. Boser, A. P. Pisano, "Polysilicon integrated microsystems: Technologies and applications", Sensors and Actuators A 56, pp. 167-177, 1996.

2. S. Brown, G. C. Johnson, W. G. Knauss, W. N. Sharpe, "Round-Robin test on modulus and strength of polysilicon", MRS Proceedings 518, 1998.

3. D. Maier-Schneider, J. Malbach, E. Obermeier, "Variations in Young's modulus intrinsic stress of LPCVD polysilicon due to high-temperature annealing", J. Micromech. Microeng. 5, pp.121-124, 1995.

4. F. A. Breton, W. G. Knauss, "Error limitations in the determination of mechanical properties of thin films", Journal of Reinforced Plastic and Composites, 16 (1), 1997.

5. W. N. Sharpe, B. Yuan, R. L. Edwards, "A new technique for measuring the mechanical properties of thin films", J. of Microelectromechanical Systems 6 (3), pp. 193-199, 1997.

6. W. N. Sharpe, R. Vaidyanathan, B. Yuan, G. Bao, R. L. Edwards, "Effect of etch holes on the mechanical properties of polysilicon", J. Vac. Science Technol. B 15 (5), pp.1599-1603, 1997.

7. S. Greek, F. Ericson, S. Johansson, J. Schweitz, "In situ tensile strength measurement and Weibull analysis of thick film and thin film micromachined polysilicon structures", Thin Solid Films 292, pp. 247-254, 1997.

8. J. Koskinen, J. E. Steinwall, R. Soave, H. H Johnson, "Microtensile testing of free-standing polysilicon fibers of various grain sizes", J. Micromech. Microeng. 3, pp.13-17, 1993.

9. T. Tsuchiya, O. Tabata, J. Sakata, Y. Taga, "Tensile testing of polycrystalline silicon thin films using electrostatic force grip", T.IEE Japan 116-E (10), pp. 441-446, 1996.

10. R. Maboudian, R. T. Howe, "Critical Review: Adhesion in surface micromechanical structures", J. Vac. Sci. Technol. B 15 (1), pp. 1-20, 1997.

11. N. Tas, T. Sonnenberg, H. Jansen, R. Legtenberg, M. Elwenspoek, "Stiction in surface micromachining", j. Micromech. Microeng. 6, pp. 385-397, 1997.

12. D. Maier-Schneider, A. Koprululu, S. Ballhausen Holm, E. Obermeier, "Elastic properties and microstructure of LPCVD polysilicon films”, J. Micromech. Microeng. 6 pp.436-446, 1996.

13. P. Lange, M. Kirsten, W. Riethmuller, B. Wenk, "Thick polycrystalline silicon for surface micro-mechanical applications: Deposition, structuring and mechanical characterization”, Sensors and Actuators A 54, pp. 674-678, 1996.

14. J. A. Walker, K. J. Gabriel, M. Mehregany, "Mechanical integrity of polysilicon films exposed to hydrofluoric acid solutions", Journal of Electronic Materials 20 (9), pp. 665-670, 1991.

15. G. Vendroux, W. G. Knauss, "Submicron deformation field measurements II: Improved Digital Image Correlation," To appear in Experimental Mechanics., June 1998.

16. M. A. Sutton, W. J. Wolters, W. H. Peters, W. F. Ranson, S. R. McNeil, "Determination of displacements using an improved Digital Correlation Method," Image Vision Computing, 1 (3), pp. 133-139, 1983.

17. M. A. Sutton, M. Cheng, W. H. Peters, Y. J. Chao, S. R. McNeil, "Application of an optimized Digital Image Correlation Method to planar deformation analysis," Image Vision Computing 4 (3), pp. 143-150, 1986.

18. M. A. Sutton, S. R. McNeil, J. Jang, M. Babai, "The effect of subpixel image restauration on Digital Image Correlation estimates," Optical Engineering 27 (10), pp. 870-877, 1988.

19. P. F. Luo, Y. J. Chao, M. A. Sutton, W.H. Peters, "Accurate measurement of three-dimensional deformations in deformable and rigid bodies using computer vision," Experimental Mechanics 33 (2), pp.123-132 (1993). 